\title{
On Existence Criteria and Approximation Procedures for Integral Equations of the First Kind
}

\author{
By C. W. Groetsch
}

\begin{abstract}
The existence of solutions of Fredholm integral equations of the first kind is characterized in terms of the convergence properties of a general approximation procedure based on a spectral analysis of the integral operator. Applications are given to some iterative and regularization methods. In particular, some results of Diaz and Metcalf are generalized.
\end{abstract}

1. Introduction. Diaz and Metcalf [1] have shown that Picard's necessary and sufficient condition for the existence of a solution of a Fredholm integral equation of the first kind

$$
\int_{a}^{b} K(s, t) x(t) d s=y(t)
$$

is equivalent to the convergence of the infinite series generated by Fridman's iteration [3] with initial approximation $\mu y$, where $\mu$ is a suitable scalar. It is the purpose of this note to show that stronger results can be obtained for more general approximation procedures. The proofs of the general theorems seem to be no more difficult than the special proofs given by Diaz and Metcalf.

2. Theorems. As in [1] we shall phrase our results abstractly and consider the equation

$$
A x=y
$$

where $A$ is a compact linear operator on a real Hilbert space $H$ which we will at least initially assume to be selfadjoint and positive semidefinite. It is then well known that $A$ has a countable set of eigenvalues $\lambda_{1} \geqslant \lambda_{2} \geqslant \cdots \geqslant 0 \quad\left(\lambda_{1}>0\right)$ and a corresponding set of orthonormal eigenvectors $u_{1}, u_{2}, \ldots$. In order to have a uniform summation convention, we shall assume that $\lambda_{k}>0$ for $k=1,2, \ldots$ (in the contrary case we need only replace the infinite sums below with finite sums). Each element $x$ of $H$ then has a unique representation of the form

$$
x=x_{N}+\sum_{k=1}^{\infty} c_{k} u_{k}
$$

where $x_{N} \in N(A)=\{z \in H: A z=0\}$ and $\Sigma_{k=1}^{\infty} c_{k}^{2}<\infty$.

We will make use of the following lemma which is a straightforward modification of [1, Lemma 2].

Received November 13, 1974.

AMS (MOS) subject classifications (1970). Primary 45L05, 47A50; Secondary 65R05.

Key words and phrases. Integral equations of first kind, compact operator, existence criteria, iterative procedures, regularization. 
Lemma. Suppose $\left\{a_{t, k}: t \in D\right\}_{k=1}^{\infty}$ is a sequence of real nets with $\Sigma_{k=1}^{\infty} a_{t, k}^{2} \leqslant$ $\Gamma<\infty$ for each $t \in D$ and $\lim _{t} a_{t, k}=a_{k}$ for each $k$ where $\Sigma_{k=1}^{\infty} a_{k}^{2}<\infty$. If $x_{t}=$ $\Sigma_{k=1}^{\infty} a_{t, k} u_{k}$, then $\lim _{t} x_{t}=\Sigma_{k=1}^{\infty} a_{k} u_{k}$.

The reader who is unfamiliar with the terminology of nets may without essential loss of understanding consider $D$ to be either the set of positive integers (which is the case considered in [1, Lemma 2]) or the set of positive real numbers. In the applications given below, these are the only directed sets $D$ which are used. The limit $\lim _{t}$ is to be interpreted as $\lim _{t \rightarrow \infty}$ in either case.

Since $\left\{a_{t, k}\right\}_{k=1}^{\infty}$ is square summable for each $t \in D$, the vector $x_{t}$ defined in the Lemma above exists for each $t \in D$. The proof of the Lemma now proceeds by considering $x_{t}-x$ where

$$
x=\sum_{k=1}^{\infty} a_{k} u_{k}
$$

and showing that $\left\{x_{t}-x\right\}$ converges to zero in essentially the same way that $\left\{x_{n}\right\}$ of [1, Lemma 2] is shown to converge strongly to zero.

We will consider approximation procedures of the following form. Let $\left\{\phi_{t}: t \in D\right\}$ be a net of continuous real-valued functions defined on some interval containing $\left[0, \lambda_{1}\right]$ such that $\left\{\lambda \phi_{t}(\lambda)\right\}$ is uniformly bounded and $\lim _{t} \phi_{t}\left(\lambda_{k}\right)=\lambda_{k}^{-1}$ for each $k$. If $\phi_{t}(A)$ is the operator associated with $\phi_{t}$ by the way of the spectral theorem for selfadjoint operators $\left[4\right.$, p. 351] then $\phi_{t}(A) y$ is an approximation to a solution of $(2.1)$ if such a solution exists.

THEOREM 1. If $x$ is a solution of (2.1), then $y \perp N(A)$ and $\lim \phi_{t}(A) y=x-x_{N}$.

Proof. That $y \perp N(A)$ follows since the range of $A$ is contained in $N(A)^{\perp}[4, \mathrm{p}$. 250]. If $A x=y$ and $x=x_{N}+\Sigma_{k=1}^{\infty} c_{k} u_{k}$, then

$$
\phi_{t}(A) y=\phi_{t}(A) A x=\sum_{k=1}^{\infty} c_{k} \phi_{t}(A) \lambda_{k} u_{k}=\sum_{k=1}^{\infty} c_{k} \lambda_{k} \phi_{t}\left(\lambda_{k}\right) u_{k} .
$$

Since $\left\{\lambda \phi_{t}(\lambda)\right\}$ is uniformly bounded on $\left[0, \lambda_{1}\right]$, we may apply the Lemma with $a_{t, k}=$ $c_{k} \lambda_{k} \phi_{t}\left(\lambda_{k}\right)$ and $a_{k}=c_{k}$ to obtain

$$
\lim _{t} \phi_{t}(A) y=\sum_{k=1}^{\infty} c_{k} u_{k}=x-x_{N}
$$

A formally stronger converse holds.

THEOREM 2. If $y \perp N(A)$ and $\left\{\phi_{t}(A) y\right\}$ has a weak limit point $x$, then $A x=y$, $x_{N}=0$ and $x=\lim _{t} \phi_{t}(A) y$.

Proof. Suppose that $x$ is the weak limit of a subnet $\left\{\phi_{t^{\prime}}(A) y\right\}$. Then $A \phi_{t^{\prime}}(A) y$ converges strongly to $A x$ since $A$ is compact [4, p. 287]. Since $y \perp N(A)$, there is a square-summable sequence $\left\{d_{k}\right\}$ such that $y=\Sigma_{k=1}^{\infty} d_{k} u_{k}$, hence

$$
A \phi_{y^{\prime}}(A) y-y=\sum_{k=1}^{\infty}\left(1-\lambda_{k} \phi_{t^{\prime}}\left(\lambda_{k}\right)\right) d_{k} u_{k} \text {. }
$$

An application of the Lemma with $a_{t, k}=\left(1-\lambda_{k} \phi_{t^{\prime}}\left(\lambda_{k}\right)\right) d_{k}$ shows that this net con- 
verges strongly to 0 and therefore $A x=y$. Also, by Theorem $1, \lim _{t} \phi_{t}(A) y=x-x_{N}$.

Since $\left\{\phi_{t^{\prime}}(A) y\right\}$ converges weakly to $x$, it follows that $x_{N}=0$ and $\lim _{t} \phi_{t}(A) y=x$, completing the proof.

Since bounded sets in Hilbert space are weakly compact [4, p. 229], the above results show that the approximations are somewhat unequivocal; they either converge or are unbounded. In the case in which $A$ is only assumed to be compact we may prove analogous theorems as in [1] by considering instead of (2.1) the equation $A^{*} A x$ $=A^{*} y$ where $A^{*}$ is the adjoint of $A$.

3. Applications. We now give some applications by making choices for the net $\left\{\phi_{t}\right\}$. Below $\mu$ will denote a scalar such that $0<\mu<2 / \lambda_{1}$.

One way to approximate $\lambda^{-1}$ is by viewing it as a fixed point of the contractive function $f(x)=(1-\mu \lambda) x+\mu$ and using the successive approximations

$$
\phi_{0}(\lambda)=\mu, \quad \phi_{n+1}(\lambda)=(1-\mu \lambda) \phi_{n}(\lambda)+\mu .
$$

The sequence $\left\{\phi_{n}\right\}$ then satisfies the requirements of the previous section. If $x_{n}=$ $\phi_{n}(A) y$, then $\left\{x_{n}\right\}$ is the sequence generated by Fridman's iteration [3] with $x_{0}=\mu y$ and the theorems give a generalization of $\left[1\right.$, Theorem $\left.\mathrm{a}_{1}\right]$.

We now give three other examples of nets which may be used in the theorems above. If $\phi_{0}(\lambda)=\mu$ and

$$
\phi_{n}(\lambda)=\frac{2 n}{n+1}\left[(1-\mu \lambda) \phi_{n-1}(\lambda)+\mu\right]-\frac{n-1}{n+1} \phi_{n-2}(\lambda),
$$

then setting $\epsilon_{n}(\lambda)=\phi_{n}(\lambda)-\lambda^{-1}$, it can be shown that

$$
\epsilon_{n}(\lambda)=\frac{\mu-\lambda^{-1}}{n+1} U_{n}(1-\mu \lambda)
$$

where $U_{n}(t)$ is a Chebyshev polynomial of the second kind (see [2, p. 521]). It follows that $\left\{\phi_{n}\right\}$ possesses the properties required above. The resulting iterative method given by $x_{0}=\mu y$ and

$$
x_{n}=\frac{2 n}{n+1}\left[x_{n-1}-\mu A x_{n-1}+\mu y\right]-\frac{n-1}{n+1} x_{n-2}
$$

is D. K. Faddeev's universal algorithm with Chebyshev polynomials of the second kind (originally given for matrix equations).

The reciprocal of $\lambda$ may also be regarded as the root of the function $f(x)=$ $x^{-1}-\lambda$. Newton's method applied to this function yields a convergent sequence given by $\phi_{0}(\lambda)=\mu, \phi_{n+1}(\lambda)=\left(2-\phi_{n}(\lambda) \lambda\right) \phi_{n}(\lambda)$.

The results of the previous section are then applicable to the sequence $x_{n}=A_{n} y$ where $A_{0}=\mu I, A_{n+1}=2 A_{n}-A_{n} A A_{n}$.

As a final example we may consider the functions $\phi_{t}(\lambda)=(\lambda+t)^{-1} \quad(t>0)$. The resulting approximation procedure given by $x_{t}=(A+t I)^{-1} y$ is a Tihonov-type "regularization of order zero" (see [5, p. 1625]).

The author wishes to thank Dr. Bill Perry for providing him with a translation of [3]. 
Departinent of Mathematics University of Rhode Island Kingston, Rhode Island 02881

1. J. B. DIAZ \& F. T. METCALF, “On iteration procedures for equations of the first kind, $A x=y$, and Picard's criterion for the existence of a solution," Math. Comp., v. 24, 1970, pp. 923-935. MR 43 \#7094.

2. D. K. FADDEEV \& V. N. FADDEEVA, Computational Methods of Linear Algebra, Fizmatgiz, Moscow, 1960; English transl., Freeman, San Francisco, Calif., 1963. MR 28 \#1742; \#4659.

3. V. M. FRIDMAN, "Method of successive approximations for a Fredholm integral equation of the first kind," Uspehi Mat. Nauk, v. 11, 1956, no. 1 (67), pp. 233-234. (Russian) MR $17,861$.

4. A. E. TAYLOR, Introduction to Functional Analysis, Wiley, New York; Chapman \& Hall, London, 1958. MR 20 \#5411.

5. A. N. TIHONOV, "Regularization of incorrectly posed problems," Dokl. Akad. Nauk SSSR, v. 153, 1963, pp. 49-52 = Soviet Math. Dokl., v. 4, 1963, pp. 1624-1627. MR 28 \#5577. 